\title{
Editorial
}

\section{The fifth UK Astrobiology Conference (ASB5)}

\author{
Charles Cockell, Mark Burchell and Zita Martins
}

\section{January 2014}

Astrobiology has a successful heritage in the United Kingdom and this was nowhere more clearly demonstrated than at the Fifth UK Astrobiology Conference (ASB5) hosted in Edinburgh in April 2013. The conference was the Tenth Anniversary of the founding of the Astrobiology Society of Britain, which began at the UK's First Astrobiology Conference in Cambridge in 2003. The Society was the offspring of the UK Astrobiology Forum and Network, which first brought together the nascent UK astrobiology community. The collection of papers presented here are a few of the many contributions that were made in Edinburgh at ASB5 and attest to the huge diversity of research occurring in the UK in this field. Research in the UK currently covers the full range of subjects relevant to astrobiology including investigations on the origins of life, the habitability of other planets, both in our Solar System and orbiting other stars, and the robotic and human exploration of other planets. The activity mirrors similar lines of research being pursued by astrobiology organizations around the world.

The conference had ten sessions over its $2^{1 / 2}$ days with 34 speakers and a poster session with 49 posters on the first evening. Sessions included those focusing on Hydrocarbons and Extraterrestrial Environments, Exoplanets and Habitability, Life in Extreme Environments, Analogue Environments, Space Instrumentation and Mars Missions. Among the delegates we were happy to have significant overseas involvement with nine invited overseas speakers: Jennifer Biddle (USA), Lynn Rothschild (USA), Rene Demets (ESA, Netherlands), David Holmes (Chile), Sam Kouvanes (USA), Gerda Horneck (Germany), Frances Westall (France), Dirk Schulze-Makuch (USA) and Gordon Osinski (Canada).

The 2013 conference was also the occasion of the inauguration of the UK Centre for Astrobiology (UKCA), which was founded in 2011 in Edinburgh. During the evening before the conference a series of lectures at the Scottish National Museum was held for this occasion. The Head of the College of Science and Engineering, University of Edinburgh, Professor Lesley Yellowlees and the Head of the School of Physics and Astronomy, University of Edinburgh, Professor Arthur Trew gave introductions followed by an excellent presentation on astrobiology by Dr Lynn Rothschild, NASA Ames Research Centre, USA on 'Life in Extreme Environments'. The UKCA provided a summary of its intentions over the coming years. We were then treated to an inspired public lecture by Dr Christopher McKay, also of NASA Ames Research Centre, on 'The Search for Life Beyond the Earth'.

The UKCA plans to contribute to astrobiology activity by a variety of means, including its development of a subsurface astrobiology laboratory and analogue science activity at Boulby Mine, the construction of new extraterrestrial simulation facilities and outreach activities through its astrobiology summer academy. Along with the ASB, the UKCA is the second UK organization affiliated with the NASA Astrobiology Institute as an affiliate member. The UKCA was conceived as a 'virtual' centre managed in Edinburgh, but with the goal of putting together 'real' facilities (such as the subsurface laboratory) when it can. Its structure is not dogmatically fixed and in 2014 we will review it.

It was fitting that this event occurred at the same time as the Astrobiology Society of Britain Conference. Ten years after the founding of the society, it marked the continuation, and development of, astrobiology activity in the UK among many other astrobiology activities occurring at other 
UK Universities. Science is not a zero-sum game and the more centres, societies and organizations that can be generated to advance any science, in this case astrobiology, the better.

The scientific discussions we had during the 3-day conference were, of course, fuelled by a whisky and gin tasting poster session. 'Old Spaceside' was a pioneering experiment in forming our own brand of astrobiology-themed whisky. The results of this experiment remain highly inconclusive. The conference ceilidh brought the attendees together in a chaotic and wonderful finale.

The number of attendees that came to the conference (142) was almost double the number at the first conference in 2003. Over the next 10 years there is every reason to suspect that with leadership of the Astrobiology Society of Britain, this number should grow further. With new missions to Mars, to comets and icy moons, and the construction of new telescopes capable of detecting rocky planets around distant stars, the UK Astrobiology Conference of 2023 should be an excellent event, socially and scientifically, in our quest to understand the origin, evolution and distribution of life in the Universe. 